\title{
Freedericksz Transition in Hybrid Aligned Nematic Liquid Crystal Cell*
}

\author{
G. Barbero \\ CNR-NATO Grantee c/o, Laboratoire de Physique des Solides, Université de Paris-Sud, \\ Bât. $510-91405$ Orsay (France)
}

Z. Naturforsch. 39 a, 575-583 (1984); received February 11, 1984

\begin{abstract}
We analyse the Freedericksz transition and the flexoelectric deformation in a hybrid aligned nematic liquid crystal cell. We show that the threshold field for the first effect is smaller than that for a homogeneous sample.

The calculations are made in the one constant approximation and in the general situation.

Furthermore, in the adiabatic approximation we calculated the polarization plane rotation vs. the applied magnetic field, in the one constant approximation. A similar calculation is made for the flexoelectric effect. In particular we show that it is possible to extend the analysis to a situation of weak anchoring energy.
\end{abstract}

\section{Introduction}

It is well known that a nematic liquid crystal oriented uniformly parallel to the limiting plates (planar samples) will undergo a Freedericksz transition in a magnetic (or electric) field applied parallel to the boundaries and perpendicular to the initial direction [1].

In this case the deformation is a pure twist, and the threshold field is given by $H_{\mathrm{c}}=(\pi / d) \sqrt{K_{22} / \chi_{\mathrm{a}}}$. If the sample is in homeotropic configuration the same magnetic field gives a distortion only if $H>H_{\mathrm{c}}=(\pi / d) \sqrt{K_{33} / \chi_{\mathrm{a}}}$, and the deformation, in this situation, is a splay + bend.

At the present time liquid crystal cells employing this effect, are widely used for making devices to display information or to regulate optical transparencies, and in many other devices.

Deuling [2] has studied the Freedericksz transition of nematic in an oblique magnetic field, and has shown that the threshold field is a function of the angle formed by $\boldsymbol{H}$ with the normal to the bounding plates.

He also studied $[3,4]$ the deformation of nematic liquid crystal cell in a crossed electric and magnetic fields. In this case the first gives, above the threshold, a deformation (for example splay + bend); the

\footnotetext{
* PACS 61.30-v; partially supported by GNSM of Italian CNR through a coordinate contract with Calabria University.

Reprint requests to G. Barbero, Dipartimento di Fisica, Politecnico di Torino, C. so Duca degli Abruzzi, 24 10129 Torino, Italy.
}

second one, above a threshold depending on the preexistent distortions (i.e. on the first field), gives a twist deformation.

As shown in $[3,4]$ the problem is very difficult mathematically, and only a numerical procedure gives some information on the threshold fields, also in the one constant approximation.

This fact is a consequence of the very general distortion (splay + bend + twist), governed by two coupled second order differential equation, that are of the Sturn-Liouville kind near the threshold [3, 4].

The present investigation tries to obtain quantitative results in the case where $\boldsymbol{H}$ is still perpendicular to the initial distortion of a hybrid nematic liquid crystal cell.

This type of cell was recently analyzed by Durand's group $[5,6]$ for flexoelectric properties and by Unical's group [7,8] for non linear optical properties.

In this cell the second order phase transition taking place at the critical field is associated with the initial symmetry of $\boldsymbol{n}$ with respect to $\boldsymbol{H}$ and, then the symmetry structure of the free energy density with respect to the twist angular variable.

In the general case discussed here we will show that the critical field for a hybrid cell is smaller than the critical field for an uniform sample.

Furthermore, connection is made with the measurable quantity, i.e. the rotation of polarisation plane, mainly with the distorting field. A knowledge of the mechanism governing this quantity is also of interest for possible application of the hybrid nematic cell in display technology. 


\section{The Euler-Lagrange Equations}

Here we consider how an external magnetic field influences the director configuration in a thin layer of nematic liquid crystal, bounded by two plane solid boundaries giving one the planar and the other the homeotropic orientation. Without external field we have the so-called hybrid orientation [5] (Figures 1,2).

It is convenient to choose a set of cartesian coordinates $(x, y, z)$ so that the solid boundaries occupy the planes $x=0$ (planar) and $x=d$ (homeotropic), and such that the orientations on the first and second plane are parallel to the $y$ - and $x$-axis, respectively.

We examine the situation in which a uniform magnetic field $H$ is applied parallel to the bounding walls and is parallel to the $Z$-axis (see Figure 2).

It seems reasonable to consider director field of the form

$$
n_{x}=\sin \psi \cos \psi ; \quad n_{y}=\cos \psi \cos \gamma ; \quad n_{z}=\sin \psi,
$$

where $\psi$ and $\chi$ are defined in Figure 3.

In this case, by using the well known Franck's formula for the free energy density of the nematic liquid crystal [1], we have

where

$$
\begin{aligned}
F(\chi, \dot{\chi} ; \psi, \dot{\psi})= & \frac{1}{2} \Lambda_{1}(\chi, \psi) \dot{\chi}^{2}+\frac{1}{2} \Lambda_{2}(\chi, \psi) \dot{\psi}^{2} \\
& +\Lambda_{3}(\chi, \psi) \dot{\chi} \dot{\psi}+F_{\mathrm{H}}(\chi),
\end{aligned}
$$

$$
\begin{aligned}
\Lambda_{1}(\%, \psi)= & K_{11} \sin ^{2} \chi \sin ^{2} \psi \\
& +K_{22} \cos ^{2} \psi+K_{33} \cos ^{2} \chi \sin ^{2} \psi, \\
\Lambda_{2}(\%, \psi)= & K_{11} \cos ^{2} \chi \cos ^{2} \psi \\
& +K_{22} \cos ^{2} \chi \sin ^{2} \chi \sin ^{2} \psi \\
& +K_{33} \cos ^{4} \chi \sin ^{2} \psi, \\
\Lambda_{3}(\%, \psi)= & \left(K_{22}-K_{11}\right) \sin \chi \cos \chi \sin \psi \cos \psi,
\end{aligned}
$$

and $F_{\mathrm{H}}=-\frac{1}{2} \chi_{\mathrm{a}} H^{2} \sin ^{2} \chi_{\text {is }}$ the free energy density associated to the magnetic field $\left(\chi_{\mathrm{a}}=\chi_{\perp}-\chi_{\perp}\right.$ is the constant magnetic anisotropy susceptibility).

If $\chi \rightarrow 0$ from Eq. (2) we have that

$$
\begin{aligned}
& A_{1}(\psi, \psi)=i_{10}(\psi)+i_{12}(\psi) \chi^{2}+O\left(\chi^{4}\right) \\
& A_{2}(\psi, \psi)=i_{20}(\psi)+i_{22}(\psi) \chi^{2}+O\left(\chi^{4}\right), \\
& \Lambda_{3}(\%, \psi)=i_{31}(\psi)+O\left(\chi^{2}\right),
\end{aligned}
$$

where:

$$
\begin{aligned}
& \lambda_{10}(\psi)=K_{33} \sin ^{2} \psi+K_{22} \cos ^{2} \psi, \\
& \lambda_{12}(\psi)=\left(K_{11}-K_{33}\right) \sin ^{2} \psi,
\end{aligned}
$$

$$
\begin{aligned}
\lambda_{20}(\psi)= & K_{11} \cos ^{2} \psi+K_{33} \sin ^{2} \psi, \\
\lambda_{22}(\psi)= & \left(K_{22}-K_{33}\right) \sin ^{2} \psi \\
& -\left(K_{11} \cos ^{2} \psi+K_{33} \sin ^{2} \psi\right), \\
\lambda_{31}(\psi)= & \left(K_{22}-K_{11}\right) \sin \psi \cos \psi .
\end{aligned}
$$

It follows from experimental measurement that the functions $\lambda_{10}(\psi)$ and $\lambda_{20}(\psi)$ are not negative, while $\lambda_{12}(\psi)$ and $\lambda_{22}(\psi)$ are not positive. In this paper it is assumed that they are strictly positive and negative, respectively. By minimizing the total free energy we obtain the following Euler-Lagrange equations:

$$
\begin{aligned}
\Lambda_{1} \ddot{\psi}+ & \frac{1}{2} \frac{\partial \Lambda_{1}}{\partial \chi} \dot{\chi}^{2}-\frac{\partial \Lambda_{1}}{\partial \psi} \dot{\psi} \dot{\psi}+\Lambda_{3} \ddot{\psi} \\
& -\left(\frac{\partial \Lambda_{3}}{\partial \psi}+\frac{1}{2} \frac{\partial \Lambda_{2}}{\partial \chi}\right) \dot{\psi}^{2}-\frac{\partial F_{\mathrm{H}}}{\partial \chi}=0 \\
\Lambda_{2} \ddot{\psi}+ & \frac{1}{2} \frac{\partial \Lambda_{2}}{\partial \psi} \dot{\psi}^{2}-\frac{1}{2} \frac{\partial \Lambda_{1}}{\partial \psi} \dot{\chi}^{2}+\Lambda_{3} \ddot{\ddot{\psi}}=0
\end{aligned}
$$

with the boundary conditions

$$
\chi(0)=\chi(d)=0, \quad \psi(0)=0 ; \psi(d)=\pi / 2
$$

in the strong anchoring hypothesis.

In (5) and (6), the dot represents derivation with respect to the coordinate $x$. For the symmetry of the magnetic energy the $\%$-orientation is a thresholdphenomenon. Therefore there exists a critical value for the magnetic field $H_{\mathrm{c}}$, below which $\chi \equiv 0, \forall x \in$ $(0, d)$.

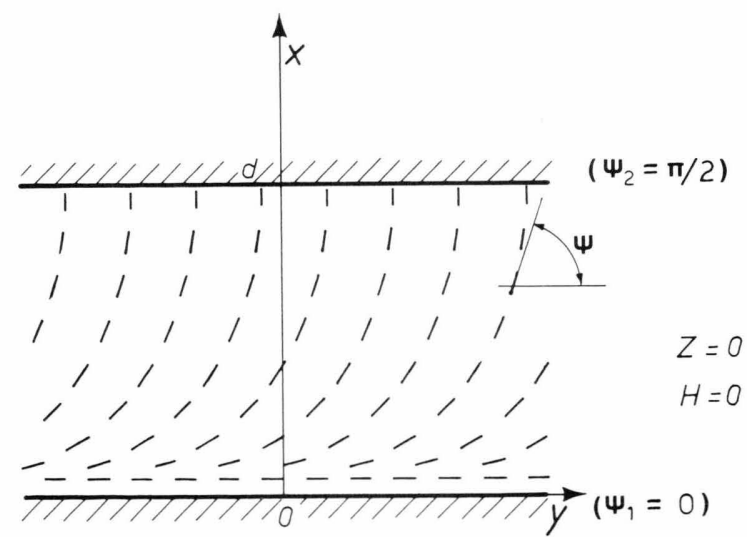

Fig. 1. Hybrid nematic liquid crystal cell, in the strong anchoring hypothesis. In a practical case the upper plate is treated with silane (homeotropic alignment), and the lower one with Keramid (planar alignment). 

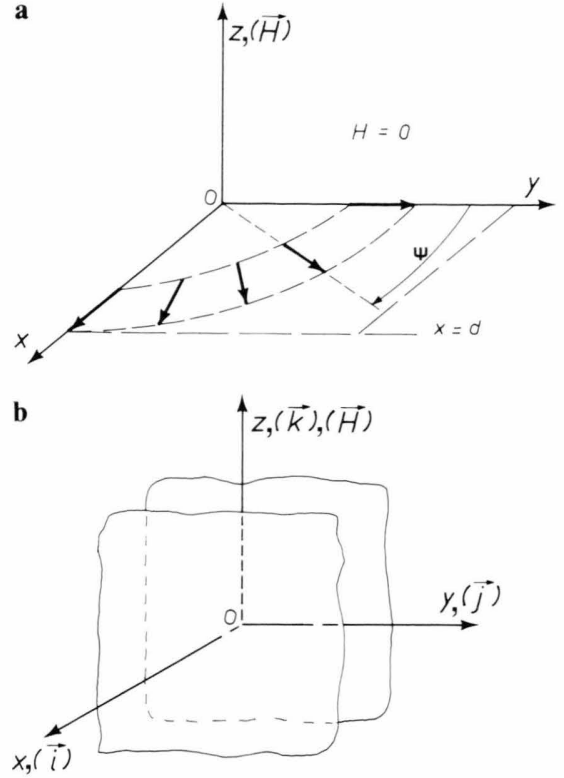

Fig. 2. Cartesian reference frame employed in the text. The magnetic field $\boldsymbol{H}$ is parallel to $\boldsymbol{Z}$-axis (a). Surfaces limiting the sample (b). The $\psi$-angle is related to the splay + bend deformation.

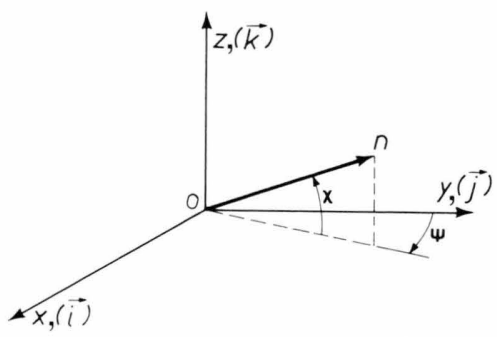

Fig. 3. Angular coordinates of the nematic director $\boldsymbol{n}$ in the hypothesis of uniform treatment of the surfaces $\psi=\psi(x)$ and $\chi=\chi(x)$ only. The $\chi$ angle is related to the twist deformation.

Then for $H \rightarrow H_{\mathrm{c}}^{+}$we have $\chi \rightarrow 0$. In this limit, by using (3), Eqs. (5) and (6) become

$$
\begin{gathered}
D\left[\lambda_{10}(\psi) \dot{\chi}\right]=\left[\left(\lambda_{22}-\lambda_{31}^{\prime}\right) \dot{\psi}^{2}-\lambda_{31} \ddot{\psi}-\chi_{\mathrm{a}} H^{2}\right] \chi, \\
\lambda_{20}(\psi) \dot{\psi}^{2}=\alpha^{2},
\end{gathered}
$$

where $D=\mathrm{d} / \mathrm{d} x$ and ${ }^{\prime}=\mathrm{d} / \mathrm{d} \psi$. In (9) $\alpha$ is an integration constant determined by the boundary conditions (7).

Equations (8) and (9) are the general equations, valid only for $H \rightarrow H_{\mathrm{c}}^{+}$.

\section{II.1. One constant approximation}

First we examine the one constant approximation, which is not very realistic, but allows us to make a semiquantitative estimate of the threshold. In this case $\left(K_{11}=K_{22}=K_{33}\right)$ we have $i_{10}^{*}=K, \quad i_{12}^{*}=0$, $i_{20}^{*}=K, i_{22}^{*}=-K, \lambda_{31}^{*}=0$, and the Eqs. (5) and (6) have the first integrals

$$
\begin{aligned}
& \dot{\chi}^{2}+\cos ^{2} \chi \dot{\psi}^{2}+\zeta^{-2} \sin ^{2} \chi=a^{2}, \\
& \cos ^{2} \chi \dot{\psi}=b
\end{aligned}
$$

as it is easy to show with symmetry considerations [9].

Equations (10) and (11) are valid for any field $H$.

In (10) and (11), $a$ and $b$ are two integrations constants, that are determined by the boundary conditions (7), and $\zeta$ is the coherence length $\left(\zeta^{2} H^{2}=K / \gamma_{\mathrm{a}}\right)$.

Utilizing (11), (10) becomes

$$
\dot{\gamma}^{2}+\left(b^{2} / \cos ^{2} \chi\right)+\zeta^{-2} \sin ^{2} \chi=a^{2} .
$$

Therefore

$$
\begin{array}{r}
\dot{\chi}= \pm\left\{\left[\left(b / \cos \chi \cos \chi_{\mathrm{mx}}\right)^{2}+\zeta^{-2}\right]\right. \\
\left.\cdot\left(\sin ^{2} \chi_{\mathrm{mx}}-\sin ^{2} \chi\right)\right\}^{1 / 2}
\end{array}\left\{\begin{array}{l}
+ \text { for } \chi \in\left(0, \chi_{\mathrm{mx}}\right), \\
- \text { for } \chi \in\left(\chi_{\mathrm{mx}}, 0\right) .
\end{array}\right.
$$

Consequently

$$
\begin{aligned}
& \int_{0}^{\chi_{\mathrm{mx}}}\left\{\left[\left(b / \cos \chi \cos \chi_{\mathrm{mx}}\right)^{2}+\zeta^{-2}\right]\right. \\
& \left.\cdot\left(\sin ^{2} \chi_{\mathrm{mx}}-\sin ^{2} \chi\right)\right\}^{-1 / 2} \mathrm{~d} \chi=d / 2 .
\end{aligned}
$$

With the change of variable $\sin \mu=\sin \chi / \sin \chi_{\mathrm{mx}}$ we rewrite (13) as

$$
\int_{0}^{\pi / 2} f_{1}\left(\mu ; \chi_{\mathrm{mx}}\right) \mathrm{d} \mu=d / 2,
$$

where

$$
\begin{aligned}
f_{1}\left(\mu ; \gamma_{\mathrm{mx}}\right)= & \left\{\left(b / \cos \chi_{\mathrm{mx}}\right)^{2}\right. \\
& \left.+\zeta^{-2}\left(1-\sin ^{2} \chi_{\mathrm{mx}} \sin ^{2} \mu\right)\right\}^{-1 / 2} .
\end{aligned}
$$

In the limit $\chi_{\mathrm{mx}} \rightarrow 0$ we deduce the critical field (via the coherence length $\zeta$ )

$$
\zeta_{\mathrm{c}}^{-2}=(\pi / d)^{2}-b_{\mathrm{c}}^{2} .
$$

In order to calculate $b_{c}$ we analyze (11). After manipulation, employing (13), one obtains the following conditions

$$
\frac{\pi}{2}=2 b \int_{0}^{\pi / 2} f_{2}\left(\mu ; \chi_{\mathrm{mx}}\right) \mathrm{d} \mu,
$$

where

$f_{2}\left(\mu, \gamma_{\mathrm{mx}}\right)=f_{1}\left(\mu ; \gamma_{\mathrm{mx}}\right) /\left(1-\sin ^{2} \chi_{\mathrm{mx}} \sin ^{2} \mu\right)$. 
Equation (17) in the limit $\%_{\mathrm{mx}} \rightarrow 0$ gives

$$
\frac{\pi}{2}=\pi b_{c} / \sqrt{b_{c}^{2}+\zeta_{c}^{-2}}
$$

(assuming strong anchoring hypothesis $\psi_{2}=\pi / 2$ and $\left.\psi_{1}=0, \forall H\right)$.

In this event, from (16) and (18) we have $b_{\mathrm{c}}=\pi / 2 d$ and consequently $\zeta_{c}^{-2}=(3 / 4)(\pi / d)^{2}$. Then the critical field is

$$
H_{\mathrm{c}}=\frac{\pi}{d} \sqrt{\frac{3}{4} \frac{K}{\chi_{\mathrm{a}}}} .
$$

Therefore $H_{\mathrm{c}}$ (hybride) $<H_{\mathrm{c}}^{(\mathrm{F})}$ (homogeneous) [1].

\section{II.2. General case}

In the one constant approximation we have determined the threshold field by using the conservation laws (10), (11), but it is possible to calculate this field in an other way, i.e. by analyzing the total free energy [2]. In the one constant approximation (1) is

$$
F=\frac{1}{2} K\left\{\dot{\chi}^{2}+\cos ^{2} \chi \dot{\psi}^{2}-\zeta^{-2} \sin ^{2} \chi\right\} .
$$

By putting $\chi=\sum_{n=0}^{\infty} \chi_{(\mathrm{n})}$ and $\psi=\sum_{n=0}^{\infty} \psi_{(n)}$, where $\chi_{(0)} \equiv 0$ and $\psi_{(0)}=\frac{\pi}{2 d} x$, and furthermore $\chi_{(n)}$ and $\psi_{(n)}$ being of the same order, from (20) we deduce

$$
\begin{aligned}
\Delta F=F- & F(H=0)=\frac{1}{2} K\left\{\frac{\pi}{d} \dot{\psi}_{(1)}\right. \\
+ & {\left.\left[\dot{\gamma}_{(1)}^{2}+\dot{\psi}_{(1)}^{2}-\left(\left(\frac{\pi}{2 d}\right)^{2}+\zeta^{-2}\right) \chi_{(1)}^{2}\right]\right\}+\ldots }
\end{aligned}
$$

From (21) we have that the first order EulerLagrange equation is identically satisfied, while the second order one gives: $\psi_{(1)}=A x+B$, with the boundary conditions $\psi_{(1)}(0)=\psi_{(1)}(d)=0$. Then $A=B=0 \Rightarrow \psi_{(1)} \equiv 0$ (this conclusion is valid also in the case of weak anchoring energy).

Therefore we obtain

$$
\Delta F=\frac{1}{2} K\left\{\dot{\gamma}_{(1)}^{2}-\left[\left(\frac{\pi}{2 d}\right)^{2}+\zeta^{-2}\right] \chi_{(1)}^{2}\right\}+\ldots
$$

If we consider a $\chi$-distortion of the kind $\chi_{(1)}=$

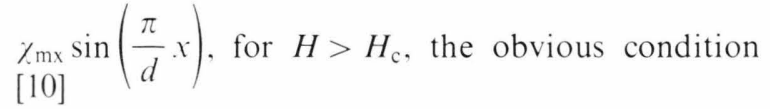

$$
\Delta F=\int_{0}^{d} \Delta F \mathrm{~d} x<0
$$

gives the relation (19).
This procedure is applicable to determine the threshold field in the general case $\left(K_{11} \neq K_{22} \neq K_{33}\right)$, where the nonlinear equations (5), (6) are quite complicated and it is not possible to give an explicit form at $\chi$ and $\psi$ (as in (13), (14) and (11), (17)).

From (1)-(4) it may be shown that the energy difference $\Delta F$ is given by

$$
\begin{aligned}
\Delta F= & \frac{1}{2}\left\{2 \lambda_{20}\left(\psi_{(0)}\right) \dot{\psi}_{(0)} \dot{\psi}_{(1)}\right. \\
& \left.+\left(K_{33}-K_{11}\right) \sin 2 \psi_{(0)} \dot{\psi}_{0}^{2} \psi_{1}\right\} \\
+ & \frac{1}{2}\left\{\lambda_{20}\left(\psi_{(0)}\right) \dot{\psi}_{1}^{2}+\lambda_{10}\left(\psi_{(0)}\right) \dot{\chi}_{(1)}^{2}\right. \\
& \quad+\lambda_{22}\left(\psi_{(0)}\right) \dot{\psi}_{(0)}^{2} \chi_{(1)}^{2} \\
& \left.+2 \lambda_{31}\left(\psi_{(0)}\right) \chi_{(1)} \dot{\psi}_{(1)} \dot{\psi}_{(0)}-\chi_{\mathrm{a}} H^{2} \chi_{(1)}^{2}\right\}+\ldots,
\end{aligned}
$$

where $F(H=0)=\frac{1}{2} \lambda_{20}\left(\psi_{(0)}\right) \dot{\psi}_{(0)}^{2}$.

By using (24) we have for the first order that $\psi_{(0)}$ is the solution of (9).

At the second order we obtain

$$
\dot{\psi}_{(1)}=\beta / i_{20}\left(\psi_{(0)}\right),
$$

where $\beta$ is another integration constant. Equation (25) gives, by using (9),

$$
\psi_{(1)}=(\beta / \alpha) \int_{0}^{\psi_{00}(x)} \lambda_{20}^{-1 / 2}(\mu) \mathrm{d} \mu+\text { const. }
$$

But $\psi_{(0)}(0)=0, \psi_{(0)}(d)=\pi / 2$ and $\psi_{(1)}(0)=\psi_{(1)}(d)$ $=0$. Consequently $\beta=$ const $=0 \Rightarrow \psi_{(1)} \equiv 0, \forall x \in$ $(0, d)$.

Then it follows that relation (24) becomes

$$
\begin{aligned}
\Delta F= & \frac{1}{2}\left\{\lambda_{10}\left(\psi_{(0)}\right) \dot{\chi}_{(1)}^{2}+\lambda_{22}\left(\psi_{(0)}\right) \dot{\psi}_{(0)}^{2} \chi_{(1)}^{2} \quad(27)\right. \\
& \left.+2 \lambda_{31}\left(\psi_{(0)}\right) \chi_{(1)} \dot{\psi}_{(1)} \psi_{(0)}-\chi_{\mathrm{a}} H^{2} \chi_{(1)}^{2}\right\}+\ldots .
\end{aligned}
$$

In $(27) \psi_{(0)}$ is solution of (9), and then it is given by

$$
\int_{0}^{\left.\psi_{10}\right)} \sqrt{\lambda_{20}(\psi)} \mathrm{d} \psi=\alpha \cdot x,
$$

where

$$
\alpha=\frac{1}{d} \int_{0}^{\pi / 2} \sqrt{\lambda_{20}(\psi)} \mathrm{d} \psi .
$$

In this case the condition (23), by using (27), (28) and (29) gives

$$
\begin{aligned}
\frac{1}{2} \chi_{\mathrm{a}} H^{2} & \geqq \frac{\pi}{d}\left\langle i_{10}\left(\psi_{(0)}\right) \cos ^{2}\left(\frac{\pi}{d} x\right)\right\rangle \\
& +\alpha^{2}\left\langle\frac{\lambda_{22}\left(\psi_{(0)}\right)}{i_{20}\left(\psi_{(0)}\right)} \sin ^{2}\left(\frac{\pi}{d} x\right)\right\rangle \\
& +\alpha \frac{\pi}{d}\left\langle\frac{i_{31}\left(\psi_{(0)}\right)}{i_{20}\left(\psi_{(0)}\right)} \sin \left(2 \frac{\pi}{d} x\right)\right\rangle,
\end{aligned}
$$


where $\langle G\rangle=(1 / d) \int_{0}^{d} G \mathrm{~d} x$. Relation (30) is useful to determine the threshold field in the general case.

Now it is possible to consider some particular cases of (30).

For $\psi_{(0)}=\pi / 2, \forall x \in(0, d),(30)$ gives

$$
H_{\mathrm{c}}^{(\mathrm{B})}=\frac{\pi}{d} \sqrt{\frac{K_{33}}{\chi_{\mathrm{a}}}},
$$

which is correct the deformation being a pure bend, while for $\psi_{(0)}=0, \forall x \in(0, d)$ the same equation gives

$$
H_{\mathrm{c}}^{(\mathrm{T})}=\frac{\pi}{d} \sqrt{\frac{K_{22}}{\chi_{\mathrm{a}}}}
$$

because in this case the deformation is a pure twist.

In the event that $\psi_{(0)}=\hat{\alpha}, \forall x(0, d)$, we obtain [11]

$$
H_{\mathrm{c}}^{(\alpha)}=\frac{\pi}{d} \sqrt{\frac{K_{33} \sin ^{2} \hat{\alpha}+K_{22} \cos ^{2} \hat{\alpha}}{\chi_{\mathrm{a}}}} .
$$

Finally, when $\psi_{(0)}(0)=0, \psi_{(0)}(d)=\pi / 2$ and $K_{11}=$ $K_{33} \neq K_{22}$ (two constants approximation) we have

$$
H_{\mathrm{c}}=\frac{\pi}{d} \sqrt{\frac{K_{11}+5 K_{22}}{8 \chi_{\mathrm{a}}}} \text {. }
$$

Obviously (34) gives (19) in the one constant approximation.

\section{Determination of physical observables}

We now proceed with the calculation of the angular dependence of $\chi_{\mathrm{mx}}$ on the magnetic field, in the one constant approximation. Furthermore we will determine the rotation of the polarisation plane, when the incident beam is polarized parallel to the molecular orientation on the wall at $x=0$, and the wave vector is parallel to the $x$-axis.

In order to determine $\chi_{\mathrm{mx}}$ vs $H$, for $H>H_{\mathrm{c}}$, we observe that by putting $b=b_{\mathrm{c}}+\varepsilon \chi_{\mathrm{mx}}^{2}+O\left(\chi_{\mathrm{mx}}^{3}\right)$, where $b_{\mathrm{c}}=\pi / 2 d,\left(15^{\prime}\right)$ and $\left(17^{\prime}\right)$ yield

$$
\begin{aligned}
f_{1}\left(\mu ; \chi_{\mathrm{mx}}\right) \cong & \left\{1-\frac{1}{2} \frac{b_{\mathrm{c}}^{2}+2 \varepsilon b_{\mathrm{c}}-\zeta^{-2} \sin ^{2} \mu}{b_{\mathrm{c}}^{2}+\zeta^{-2}} \chi_{\mathrm{mx}}^{2}\right\} \\
& \cdot\left(b_{\mathrm{c}}^{2}+\zeta^{-2}\right)^{-1 / 2}+O\left(\chi_{\mathrm{mx}}^{3}\right),
\end{aligned}
$$

$f_{2}\left(\mu ; \chi_{\mathrm{mx}}\right)$

$$
\begin{aligned}
\cong & \left\{1-\frac{1}{2} \frac{b_{\mathrm{c}}^{2}+2 \varepsilon b_{\mathrm{c}}-\left(2 b_{\mathrm{c}}^{2}+3 \zeta^{-2}\right) \sin ^{2} \mu}{b_{\mathrm{c}}^{2}+\zeta^{-2}} \chi_{\mathrm{mx}}^{2}\right\} \\
& \cdot\left(b_{\mathrm{c}}^{2}+\zeta^{-2}\right)^{-1 / 2}+O\left(\chi_{\mathrm{mx}}^{3}\right) .
\end{aligned}
$$

Consequently (15) and (17) give, after trivial integrations,

$$
\begin{aligned}
& \chi_{\mathrm{mx}}^{2}=4 \frac{b_{\mathrm{c}}^{2}+\zeta^{-2}}{\zeta^{-2}-2 b_{\mathrm{c}}\left(b_{\mathrm{c}}+2 \varepsilon\right)}\left\{\frac{d}{\pi}\left(b_{\mathrm{c}}^{2}+\zeta^{-2}\right)^{1 / 2}-1\right\}, \\
& \chi_{\mathrm{mx}}^{2}=4 \frac{b_{\mathrm{c}}^{2}+\zeta^{-2}}{3 b_{\mathrm{c}}+4 \varepsilon}\left\{\frac{\left(b_{\mathrm{c}}^{2}+\zeta^{-2}\right)^{1 / 2}}{2}-b_{\mathrm{c}}\right\} \zeta^{2} .
\end{aligned}
$$

From the previous equations we deduce $\varepsilon=-(1 / 2)$ $b_{\mathrm{c}}$, and then

$\chi_{\mathrm{mx}}^{2}=4\left(b_{\mathrm{c}}^{2}+\zeta^{-2}\right) \zeta^{2}\left\{(d / \pi)\left(b_{\mathrm{c}}^{2}+\zeta^{-2}\right)^{1 / 2}-1\right\}$.

Equation (37) in the limit of $b_{\mathrm{c}}=0$ (homogeneous sample: homeotropic or planar configuration) gives the well known [12] equation $\chi_{\mathrm{mx}}^{2}=4\left(\frac{H}{H_{\mathrm{c}}^{(\mathrm{F})}}-1\right)$.

By putting $h=H / H_{\mathrm{c}}^{(\mathrm{F})}$, where $H_{\mathrm{c}}^{(\mathrm{F})}$ is defined in [1], it is possible to rewrite (37) as

$$
\chi_{\mathrm{mx}}^{2}=\left(1+4 h^{2}\right)\left[\left(1+4 h^{2}\right)^{1 / 2}-2\right] / 2 h^{2} .
$$

With the dimensionless parameter $h$, the threshold condition (15) becomes $h_{\mathrm{c}}=\sqrt{3} / 2$.

Now let us examine the propagation of the polarized beam (incident plane polarisation parallel to $(\boldsymbol{i}, \boldsymbol{j})$ plane) through the cell.

In the hypothesis of adiabatic propagation the polarisation plane is coincident with the plane $\boldsymbol{n}(x)$, $i$. With the aim to calculate the output angle we consider the angle formed between $\boldsymbol{n}(x), \boldsymbol{i}$ and the plane $i, j$ in the limit $x \rightarrow d$. As shown in Fig. 4 we have

$$
\operatorname{tg} \alpha(x)=\operatorname{tg} \chi(x) / \cos \psi(x) .
$$

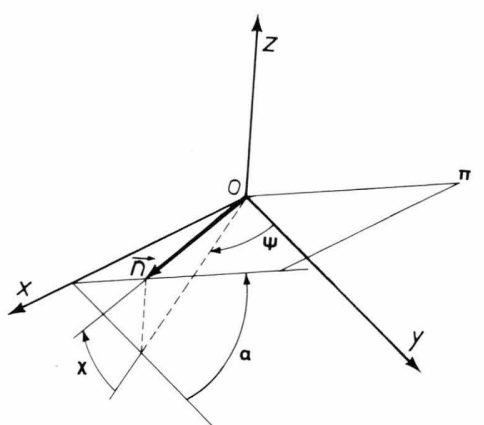

Fig. 4. Osculating plane $(\pi)$ of the $\gamma$-director line. In the adiabatic approximation the polarisation plane is coincident with the $\boldsymbol{n}(x), \boldsymbol{i}$ plane if the wave vector of the beam is parallel to $i . \alpha$ is the angle formed between the polarisation plane (at $x$ position) and the incident polarisation plane (that we suppose is parallel to the $(\boldsymbol{i}, \boldsymbol{j})$ plane). The output polarisation plane is obtained as $\lim _{x \rightarrow d} \alpha=\alpha_{\text {out }}$. 
Consequently

$$
\operatorname{tg} \alpha_{\text {out }}=\lim _{x \rightarrow d} \operatorname{tg} \alpha(x)=-\dot{\chi}(d) / \dot{\psi}(d) .
$$

By using (13) we obtain

$$
\dot{\chi}(d)=-\left[\left(b / \cos ^{2} \chi_{\mathrm{mx}}\right)^{2}+\zeta^{-2}\right]^{1 / 2} \sin \chi_{\mathrm{mx}},
$$

and then, for $h>\sqrt{3} / 2$, utilizing (38) in (40) yields

$$
\begin{aligned}
\dot{\chi}(d)= & -b_{\mathrm{c}} \frac{1+4 h^{2}}{h}\left\{\frac{\left(1+4 h^{2}\right)^{1 / 2}-2}{2}\right\}^{1 / 2} \\
& +O\left(\gamma_{\mathrm{mx}}^{2}\right) .
\end{aligned}
$$

By substituting (41) in (39) we have, finally

$$
\operatorname{tg} \alpha_{\text {out }}=\frac{1+h^{2}}{h}\left\{\frac{\left(1+4 h^{2}\right)^{1 / 2}-2}{2}\right\}^{1 / 2} .
$$

Equation (42) is plotted in Figure (5a).

It is easy to show that the polarization plane of the outing beam is parallel to the osculating plane $(\pi)$ of the director line, at $x=d$.

In fact if $x$ is the parameter of the director line $\gamma$, the parametric equations of this curve are

$$
x=x, \quad y=y(x), \quad z=z(x) .
$$
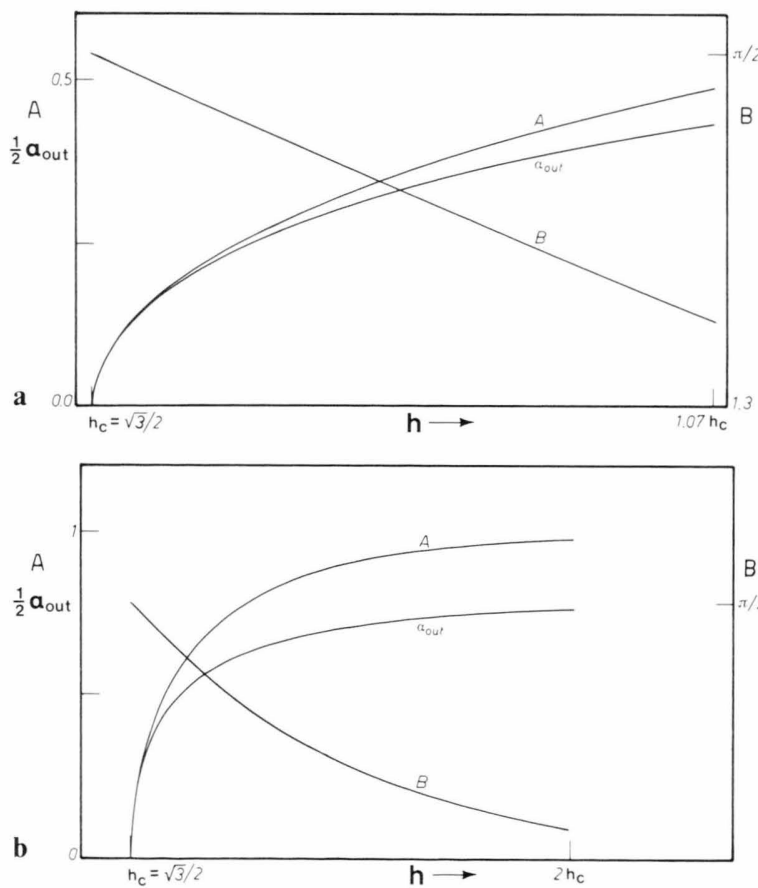

Fig. 5. Rotation of the polarisation plane vs the dimensionless magnetic field $h\left(=H / H_{\mathrm{c}}^{(\mathrm{F})}\right)$. The threshold field is $h_{\mathrm{c}}=\sqrt{3} / 2$. Behaviour near $h_{\mathrm{c}}(a)$. For any field (b). $A=\sin \% \mathrm{mx}, B=\mathrm{d} b$.
Furthermore the tangent to the $\gamma$-curve is, by definition, $\boldsymbol{n}(x)$. Then

$$
\boldsymbol{n}(x)=\frac{\mathrm{d} \boldsymbol{r}(x) / \mathrm{d} x}{\mathrm{~d} \boldsymbol{r}(x) / \mathrm{d} x},
$$

where $\quad \mathrm{d} \boldsymbol{r}(x) / \mathrm{d} x=\sqrt{1+\dot{y}^{2}(x)+\dot{z}^{2}(x)}$. From (43) we deduce

$$
\begin{aligned}
1 & =\sin \psi(x) \cos \psi(x) \sqrt{1+\dot{y}^{2}(x)+\dot{z}^{2}(x)}, \\
\dot{y}(x) & =\cos \psi(x) \cos \chi(x) \sqrt{1+\dot{y}^{2}(x)+\dot{z}^{2}(x)}, \\
\dot{z}(x) & =\sin \psi(x) \sqrt{1+\dot{y}^{2}(x)+\dot{z}^{2}(x)} .
\end{aligned}
$$

Equations (45) and (46) give, after trivial calculations,

$$
\begin{aligned}
& \dot{y}(x)=\operatorname{ctg} \psi(x), \\
& \dot{z}(x)=\operatorname{tg} \psi(x) \operatorname{cosec} \psi(x) .
\end{aligned}
$$

It is possible to integrate (47) and (48) in order to obtain the $\gamma$-curve equation.

With the aim to calculate the osculating plane equation we calculate $\ddot{y}$ and $\ddot{z}$. From (47) and (48) we have

$$
\begin{aligned}
\ddot{y}(x)= & -\dot{\psi}(x) \operatorname{cosec}^{2} \psi(x), \\
\ddot{z}(x)= & \operatorname{cosec}^{2} \psi(x) \\
& \cdot\left[\dot{\chi}(x) \sin \psi(x) \sec ^{2} \psi(x)\right. \\
& -\dot{\psi}(x) \operatorname{tg} \chi(x) \cos \psi(x)] .
\end{aligned}
$$

By using $(47)-(50)$ it is easy to obtain the osculating-plane equation by developing the determinant

$$
\left|\begin{array}{ccc}
X-x & Y-y(x) & Z-z(x) \\
1 & \dot{y}(x) & \dot{z}(x) \\
0 & \ddot{y}(x) & \ddot{z}(x)
\end{array}\right|=0,
$$

where $X, Y$ and $Z$ are the coordinates of a generic point of the osculating plane.

In particular for $x=d$, by using the boundary conditions (7), we have $\dot{y}(d)=0, \dot{z}(d)=0, \ddot{y}(d)=$ $-\dot{\psi}(d)$ and $\ddot{z}(d)=\dot{\psi}(d)$.

Consequently, (51) gives

$$
\frac{Z-z(d)}{Y-y(d)}=\operatorname{tg} \alpha_{\text {out }}=-\frac{\dot{y}(d)}{\dot{\psi}(d)},
$$

that is coincident with (39).

Equation (38) is valid only for $h \gtrsim \sqrt{3} / 2$, i.e. only near the threshold. 
For arbitrary field (39) gives, taking into account that $\dot{\psi}(d)=b$,

$$
\operatorname{tg} \alpha_{\text {out }}=\left(\frac{b^{2}}{\cos ^{2} \gamma_{\mathrm{mx}}}+\zeta^{-2}\right)^{1 / 2} \frac{\sin \gamma_{\mathrm{mx}}}{b},
$$

as plotted in Figure $5 \mathrm{~b}$ (see appendix 1).

It is very important to point out that the present calculations are made under the assumption that the pinning of the director to the walls is ideally rigid. But, by observing that the description of the phenomenon employs the $\chi$ and $\psi$ angles, that are physical angles, it is possible to examine also the weak-anchoring situation.

In the case of finite anchoring energy we have $(*)$ $\chi_{1} \neq 0, \varkappa_{2} \neq 0, \psi_{1} \neq 0$ and $\psi_{2} \neq \pi / 2$, and these angles are determined by boundary conditions involving the anchoring energy $F_{\mathrm{S}}(\chi, \psi)$.

In this hypothesis (15) and (17) become

$$
\begin{gathered}
\int_{\mu_{1}}^{\pi / 2} f_{1}\left(\mu, \chi_{\mathrm{mx}}\right) \mathrm{d} \mu+\int_{\mu_{1}}^{\pi / 2} f_{1}\left(\mu, \chi_{\mathrm{mx}}\right) \mathrm{d} \mu=d, \\
b\left\{\int_{\mu_{1}}^{\pi / 2} f_{2}\left(\mu, \chi_{\mathrm{mx}}\right) \mathrm{d} \mu+\int_{\mu_{2}}^{\pi / 2} f_{2}\left(\mu, \chi_{\mathrm{mx}}\right) \mathrm{d} \mu\right\}=\psi_{2}-\psi_{1},
\end{gathered}
$$

where $\mu_{i}=\sin ^{-1}\left(\sin \chi_{i} / \sin \chi_{\mathrm{mx}}\right)$.

Following step by step the previous procedure we obtain

$$
b_{\mathrm{c}}=\left(\psi_{2}^{0}-\psi_{1}^{0}\right) / d
$$

and

$$
\zeta_{c}^{-2}=\frac{\left[\pi-\left(\mu_{1}^{0}+\mu_{2}^{0}\right)\right]^{2}-\left[\psi_{2}^{0}-\psi_{1}^{0}\right]^{2}}{d^{2}}
$$

with $\psi_{i}^{0}=\lim _{\chi_{\mathrm{mx}} \rightarrow 0} \psi_{i}$ and $\mu_{i}^{0}=\lim _{\chi_{\mathrm{mx}} \rightarrow 0} \mu_{i}$.

Furthermore, in order to determine $\chi_{\mathrm{mx}}$ vs $H$, we deduce

$\varepsilon=-\frac{1}{2} b_{\mathrm{c}}\left\{1-\frac{1}{2} \frac{b_{\mathrm{c}}^{2}+2 \zeta^{-2}}{b_{\mathrm{c}}^{2}-\zeta^{-2}} \frac{\sin 2 \mu_{1}^{0}+\sin 2 \mu_{2}^{0}}{\pi-\left(\mu_{1}^{0}+\mu_{2}^{0}\right)}\right\}+\ldots$

and finally

The anchoring energy is contained in $\mu_{i}^{0}, \psi_{i}^{0}$ and then in $\psi_{\mathrm{mx}}$. In order to give a complete solution of the problem it is necessary to solve (10) and (11) with the boundary conditions

$$
-K \dot{\varkappa}_{1}+\left(\frac{\partial F_{\mathrm{s}}}{\partial y_{1}}\right)_{1}=0, \quad K \dot{\varkappa}_{2}+\left(\frac{\partial F_{\mathrm{s}}}{\partial \%}\right)_{2}=0
$$

and

$$
\begin{aligned}
-K \cos ^{2} \chi_{1} \dot{\psi}_{1}+\left(\frac{\partial F_{\mathrm{s}}}{\partial \psi}\right)_{1} & =0, \\
K \cos ^{2} \chi_{2} \dot{\psi}_{2}+\left(\frac{\partial F_{\mathrm{s}}}{\partial \psi}\right)_{2} & =0
\end{aligned}
$$

in the hypothesis that $F_{\mathrm{s}}=F_{\mathrm{s}}(\chi, \psi)$ only. The problem is of great interest, but nothing can be said at the present on the function $F_{\mathrm{s}}$, and then an analytical solution is not possible $[13,14]$. A study on this problem is under way and the results will be published elsewhere.

\section{Flexoelectric Effect}

Very recently Durand's group $[5,6]$ has shown that a hybrid aligned nematic cell exhibits flexoelectric properties which are very interesting.

Now we analyse the flexoelectric distortion [1].

If $\boldsymbol{E}$ is parallel the $Z$-axis the free energy density associated with the electric field is [15]

$$
\begin{aligned}
F_{\mathrm{E}}(\chi, \psi)= & \boldsymbol{E}\left(-e_{1} \boldsymbol{n} \operatorname{div} \boldsymbol{n}+e_{3} \boldsymbol{n} \times \operatorname{rot} \boldsymbol{n}\right) \\
& -\left(\varepsilon_{\mathrm{a}} / 8 \pi\right)(\boldsymbol{n} \cdot \boldsymbol{E})^{2},
\end{aligned}
$$

where $e_{i}$ are the flexoelectric coefficients and $\varepsilon_{\mathrm{a}}=\varepsilon-\varepsilon_{\perp}$ is the dielectric anisotropy susceptibility.

In these conditions (5) and (6), in the one constant approximation, become

$$
\begin{aligned}
K \ddot{\gamma} & +E\left\{\left(e_{1} \sin ^{2} \chi-e_{3} \cos ^{2} \chi\right) \cos \psi \dot{\psi}\right. \\
& \left.+(1 / 2) K \sin 2 \chi \dot{\psi}^{2}+e_{1} \cos 2 \chi \cos \psi \dot{\psi}\right\} \\
& +\left(\varepsilon_{\mathrm{a}} E^{2} / 8 \pi\right) \sin 2 \chi=0
\end{aligned}
$$

$$
\chi_{\mathrm{mx}}^{2}=4 \frac{\left[\mathrm{d}\left(b_{\mathrm{c}}^{2}+\zeta^{-2}\right)^{1 / 2}-\pi+\left(\mu_{1}^{0}+\mu_{2}^{0}\right)\right]\left(b_{\mathrm{c}}^{2}+\zeta^{-2}\right)}{\left(b_{\mathrm{c}}^{2} \frac{b_{\mathrm{c}}^{2}+2 \zeta^{-2}}{\zeta^{-2}-b_{\mathrm{c}}}+\frac{1}{2} \zeta^{-2}\right)\left(\sin 2 \mu_{1}^{0}+\sin 2 \mu_{2}^{0}\right)+\zeta^{-2}\left[\pi-\left(\mu_{1}^{0}+\mu_{2}^{0}\right)\right]} .
$$

and

From (58) it is possible to calculate the pretwist angles as $\chi_{i}=\chi_{\mathrm{mx}} \sin \mu_{i}^{0}$, and then (39) gives

$$
\operatorname{tg} \alpha_{\text {out }} \cong \frac{\operatorname{tg} \chi_{2}}{\cos \psi_{2}} \cong \chi_{\mathrm{mx}} \frac{\sin \mu_{2}^{0}}{\cos \psi_{2}^{0}} .
$$

$$
\begin{aligned}
& K \cos ^{2} \chi \ddot{\psi}-K \sin 2 \chi \dot{\chi} \dot{\psi}-e_{1} E \cos 2 \chi \dot{\chi} \cos \psi \\
& -E\left(e_{1} \sin ^{2} \chi-e_{3} \cos ^{2} \chi\right) \cos \psi \dot{\chi}=0
\end{aligned}
$$

when $\boldsymbol{E} \rightarrow 0, \chi \rightarrow 0$ and $\psi \rightarrow(\pi / 2 d) x$. By applying 
the Poincare's theorem it is possible to write

$$
\begin{aligned}
& \psi=\psi_{(0)}+\delta \psi=\sum_{n=0}^{\infty} \psi_{(n)} E^{n}, \\
& \chi=\sum_{n=0}^{\infty} \chi_{(n)} E^{n},
\end{aligned}
$$

where $\psi_{(0)}=(\pi / 2 d) x$ and $\chi_{(0)} \equiv 0$. By substituting the previous developments into (64) and (65) we obtain

$$
\begin{aligned}
\ddot{\psi}_{(0)} & =0 \ddot{\psi}_{(1)}=0, \\
\ddot{\chi}_{(1)}+ & \dot{\psi}_{(0)}^{2} \chi_{(1)}=\left(e^{* / K}\right) \cos \psi_{(0)} \dot{\psi}_{(0)}, \\
\ddot{\chi}_{(2)}+ & \dot{\psi}_{(0)}^{2} \chi_{(2)}=\left(e^{* / K}\right) \\
& \cdot\left[\cos \psi_{(0)} \dot{\psi}_{(1)}-\psi_{(1)} \dot{\psi}_{(0)} \sin \psi_{(0)}\right],
\end{aligned}
$$

where $e^{*}=e_{3}-e_{1}$.

In the strong anchoring hypothesis, (66) gives

$$
\begin{aligned}
& \psi_{(0)}=(\pi / 2 d) x ; \quad \psi_{(1)} \equiv 0, \\
& \chi_{(1)}=\left(e^{* / 2 K}\right)(x-d) \sin (\pi x / 2 d) ; \quad \chi_{(2)} \equiv 0 .
\end{aligned}
$$

Therefore

$\chi(x) \cong\left(e^{*} E / 2 K\right)(x-d) \sin (\pi x / 2 d)+O\left(E^{3}\right)$.

By putting (68) into (39) we obtain

$$
\operatorname{tg} \alpha_{\text {out }}=-\frac{e^{*} E d}{K \pi}
$$

as reported in [2].

We point out that the description employing the geometrical angle $\varphi$, as made in Durand's paper, is equivalent to that employed in the present paper, only in the strong anchoring hypothesis. In the weak anchoring situation the $\varphi$-description is not applicable, because the boundary conditions are given for the physical angles.

\section{Conclusion}

In this paper we have analyzed the Freedericksz transition and the flexoelectric deformation in an hybrid aligned nematic cell. We have shown that the threshold field for the first effect is smaller than that for a homogeneous sample. The calculations are made in the one constant approximation and in the general situation.

As in Deuling's paper, the Freedericksz threshold field depends on the initial distortion (splay + bend induced by the limiting walls).
Furthermore, in the adiabatic approximation we have calculated the polarisation plane rotation vs the applied magnetic field in the one constant approximation, and we have shown that the polarisation plane is coincident with the osculating plane of the director line. A similar calculation is made for the flexoelectric effect.

In particular we have shown that it is possible to extend the analysis to a situation of weak anchoring energy, and that it is possible, with the experiment performed in [3] or with a flexoelectric experiment, to deduce information about the general surface energy.

Acknowledgements are due to the fruitful discussions with N. V. Madhusadana (Raman Institute, Bangalore), C. Oldano (Politecnico, Torino) and $\mathrm{Ph}$. Martinot-Lagarde (Laboratoire de Physique des Solides, Orsay).

\section{Appendix 1}

In order to determine the rotation of the plane of polarization for any applied magnetic field we rewrite (13) and (17) in the following way:

$$
\begin{aligned}
& \int_{0}^{\pi / 2}\left\{\left[B^{2} /\left(1-A^{2}\right)\right]+u^{2}\left(1-A^{2} \sin ^{2} \mu\right)\right\}^{-1 / 2} \mathrm{~d} \mu= 1 / 2, \\
& B \int_{0}^{\pi / 2}\left\{\left(1-A^{2} \sin ^{2} \mu\right)^{2}\right. \text { (A.1) } \\
& \cdot\left[\left(B^{2} /\left(1-A^{2}\right)+u^{2}\left(1-A^{2} \sin ^{2} \mu\right)\right]\right\}^{-1 / 2} \mathrm{~d} \mu=\pi / 4,
\end{aligned}
$$

where the dimensionless parameters $A, B$ and $u$ are defined as

$$
A=\sin \chi_{\mathrm{mx}}, \quad B=\mathrm{d} b, \quad u=\pi h .
$$

By putting

$$
I(\omega)=\int_{0}^{\pi / 2}\left(1-\omega^{2} \sin ^{2} \mu\right)^{-1 / 2} \mathrm{~d} \mu,
$$

$J(A, \omega)=\int_{0}^{\pi / 2}\left\{\left(1-A^{2} \sin ^{2} \mu\right) \sqrt{1-\omega^{2} \sin ^{2} \mu}\right\}^{-1} \mathrm{~d} \mu$,

(A.1), (A.2) can be put in the form

$$
\begin{aligned}
& I(\omega)=\frac{1}{2}\left\{\left[B^{2} /\left(1-A^{2}\right)\right]+u^{2}\right\}^{1 / 2}, \\
& J(A, \omega)=I(\omega) \pi / 2 B
\end{aligned}
$$

if

$$
\omega^{2}=A^{2} u^{2} /\left\{\left[B^{2} /\left(1-A^{2}\right)\right]+u^{2}\right\} .
$$


From (A.5) and (A.7) we deduce

$$
\omega=A u / 2 I(\omega) .
$$

Furthermore by substituting (A.6) and (A.8) into (A.5) we derive

$$
\omega^{2}=A^{2}\left\{1-\left[\pi^{2} / 16 J^{2}(A, \omega)\left(1-A^{2}\right)\right]\right\} .
$$

With numerical calculations, (A.4) and (A.5) give $\omega$ and $A$, easily. Then (A.6) and (A.8) give $B$ and $u$, respectively.

[1] See for example: H. J. Deuling, Solid State Physics. Suppl. 14 ed. by L. Liébert, p. 77 (1978).

[2] H. J. Deuling, M. Gabay, E. Guyon, and P. Piéranski, J. Phys., Paris 36, 689 (1975).

[3] H. J. Deuling, E. Guyon, and P. Piéranski, Sol. State Comm. 15, 277 (1974).

[4] H. J. Deuling, A. Buka, and I. Janossy, J. Phys., Paris 37, 965 (1976).

[5] I. Dozov, Ph. Martinot-Lagarde, and G. Durand, J. Phys., Paris, Lett. 43, 365 (1982).

[6] I. Dozov, Ph. Martinot-Lagarde, and G. Durand, J. Phys., Paris, Lett. 44, 817 (1983).

[7] G. Barbero and F. Simoni, Appl. Phys. Lett. 41, 504 (1982).

[8] G. Barbero, F. Simoni, and P. Aiello, J. Appl. Phys. 55, 304 (1984).

[9] R. S. Akopyan and B. Ya. Zel'dovich, Zh. Eksp. Teor. Fiz. 83, 2137 (1982), Sov. Phys. JETP, 56(6), 1239 (1982).

[10] Condition (23) is similar to the condition $C<0$ in the Landau expansion $\Delta \mathscr{F}=\mathrm{C} \chi_{\mathrm{mx}}^{2}$ (see [2]).

[11] Eq. (33) is analogous to (2.12) [2], but the conditions are differents.
The functions $\gamma_{\mathrm{mx}}=\sin ^{-1} A$ and $b=B / d$ vs $h$ are drawn in Figures 5.

Figure $5 \mathrm{~b}$ shows that $\chi_{\mathrm{mx}}$ reaches $\pi / 2$ rapidly, while $b$ (related to the gradient of the splay + bend deformations) goes to zero, as expected. The rotation of the plane of polarization given by (33) is

$$
\alpha_{\text {out }}=\operatorname{tg}^{-1}\left\{\frac{2}{\pi} J(A, \omega)\right\}
$$

in this formalism. The function $\alpha_{\text {out }}=\alpha_{\text {out }}(h)$ is shown in Figure (5b).

[12] E. B. Priestly, P. J. Wojtowicz, and Ping Sheng, Introduction to liquid crystal, Plenum, New York 1975.

[13] G. Barbero, R. Bartolino, and M. Meuti, Submitted to Physica B + C (1983) and reference therein.

[14] As pointed out in [13] there is a phenomenological law to study the anchoring effect on the distortion, only if the distortion is planar (no twist). In this case the anchoring energy is modeled as $-(1 / 2) W(\boldsymbol{n} \cdot \boldsymbol{K})^{2}$, where $W$ is the anisotropy of the anchoring energy and $\boldsymbol{K}$ is the normal to the substrate (that is supposed flat). The $W$-parameter is measured by the Freedericksz threshold-method (see [13]). With the method reported in [3] it seems possible to study the $F_{\mathrm{s}}(\alpha, \psi)$ form near the equilibrium configuration. Furthermore, studying the curves $V_{\mathrm{t}}(H)$ and $V_{\mathrm{s}}(H)$ one can obtain the behaviour of $F_{\mathrm{s}}(0, \psi)$ or of $F_{\mathrm{s}}(\chi, 0)$.

[15] In (62) the dielectric term is correct only if $\varepsilon_{\mathrm{a}} \ll 1$. For the more general case (great $\varepsilon_{\mathrm{a}}$ ) see $\mathrm{H}$. J. Deuling, Mol. Cryst. Liq. Cryst. 19, 123 (1972). We point out that in Durand's experiments $\varepsilon_{\mathrm{a}} \gtrsim 1$, and then (62) is quite approximate.

[*] We employ the notations $\psi_{1}=\psi(0), \quad \psi_{2}=\psi(d)$, $\chi_{1}=\chi(0), \chi_{2}=\chi(d)$ in order to simplify $(55)-(62)$. 